\title{
Für die Einführung der Fallpauschalen (DRG) am 1. Januar 2012 ist es zu früh! - Petition an den Zentralvorstand der FMH
}

\section{Liebe Kolleginnen und Kollegen!}

Am 1. Januar 2012 soll in der Schweiz ein gegenüber dem deutschen zwar ähnliches, aber deutlich verschärftes Fallpauschalensystem eingeführt werden. Die Diskussionen zeigen immer deutlicher, dass viele Probleme noch ungelöst sind und wichtige Versprechungen der Befürworter sich schon aus heutiger Sicht nicht bewahrheiten werden. Folgende Gründe zwingen zu einem Marschhalt und zum Nachdenken, wann, in welcher Form und mit welcher Verbreitung Fallpauschalen eingeführt werden sollen:

- Wird das DRG-System ohne Änderungen eingeführt, resultieren massive Nachteile für die heute typisch gewordenen komplexen Patienten mit chronischen Erkrankungen oder Erkrankungen mehrerer Organe. Die kommenden demographischen Veränderungen lassen eine weitere $\mathrm{Zu}$ nahme dieser Patienten erwarten.

- Befürwortende Gesundheitsökonomen geben heute $\mathrm{zu}$, dass das Fallpauschalensystem - wie in Deutschland schmerzlich beobachtet - einen weiteren Kostenschub im Gesundheitswesen auslösen wird, der am intensivsten im Prämienbereich spürbar sein wird.

- Die Auswirkungen auf die ambulante, v.a. Hausarzt-Medizin sind bisher kaum absehbar. Mehraufwand für die zahlenmässig abnehmenden Grundversorger, dadurch bedingte Taxpunktsenkung und drohende Verschlechterung des Verhältnisses von Haus- und Spitalärzten, sind aber wahrscheinlich. Dies wird sich akzentuieren, wenn die DRGs auch für den ambulanten Bereich eingeführt werden.

Korrespondenz: Dr. med. Christian Hess Prof. Dr. med. Reto Krapf Dr. med. Urs Strebel reto.krapf@ksbh.ch
- Das Problem der Finanzierung der Weiterbildung (Assistenz- und Oberärzte) ist ungelöst.

- Der zunehmende ökonomische Zwang und Zeitdruck wird die Ausbildung des ärztlichen Nach- wuchses in den Spitälern und die klinische Forschung unter massiven Druck setzen.

- Die Arbeitsbelastung in den Gesundheitsberufen (namentlich auch in der Krankenpflege) wird zunehmen und für die Patienten (noch) spürbar(er) werden.

- Das vorgesehene DRG-System setzt die falschen medizinisch-ökonomischen Anreize. Gewinnbringende Leistungen werden vermehrt angeboten werden, ungeachtet ihrer medizinischen Notwendigkeit.

- Die vorgesehene, aber noch nicht etablierte «Begleit»-Forschung ist das Eingeständnis der vielen ungelösten Fragen. Warum nicht zuerst die zentralen Fragen klären helfen und dann DRG einführen?

- Viele Datenschutzprobleme sind ungelöst, der obligate Datentransfer zu den Krankenkassen ist nicht definiert und er wird ohne öffentliche Diskussion festgesetzt.

Unterstützen Sie als Ärztin oder Arzt bitte diese Petition. Wir wollen die FMH für die weitere Diskussion mit einem klaren Votum aus der Ärzteschaft unterstützen. Damit kann sie sich auch für die Verschiebung der Einführung flächendeckender Fallpauschalen einsetzen, bis die zentralen Fragen beantwortet sind. Die Zeit drängt, da der Bundesrat Ende Juni 2011 definitiv über den Einführungstermin entscheiden wird.

Für diese Anliegen können Sie und weitere interessierte, auch nicht-ärztliche Personen sich zusätzlich engagieren, wenn Sie das sog. Moratorium mitunterzeichnen (www.drg-moratorium.ch/de/).

Für das ärztliche Petitionskomitee Dr. med. Christian Hess, Dr. med. Urs Strebel, Prof. Dr. med. Reto Krapf 